\title{
CONSTRUCTION OF EXACT SOLUTION TO SINE-GORDON EQUATION ON THE BASE OF ITS CHARACTERISTIC LIE RING
}

\author{
A.V. ZHIBER, S.N. KAMAEVA
}

\begin{abstract}
In the work we consider the scheme of constructing exact solutions to the Sine-Gordon equation based on a restricting the characteristic Lie ring. We study in details the case when the dimension of the space formed by commutators of length 6 is equal to 1 .
\end{abstract}

Keywords: solitons, vector fields, Lie ring

Mathematics Subject Classification: 34A05, 35B06

\section{INTRODUCTION}

It is known that the inverse problem method of the scattering theory allows one to construct exact solutions to the Sine-Gordon equation

$$
u_{x y}=e^{u}+e^{-u}
$$

so-called solitons (see, for instance, [1], [2]).

In the present work we consider an alternative approach for constructing exact solutions to equation (1) on the base of the characteristic Lie ring.

The characteristic ring $A$ of equation (1) is generated by the vector fields (see [3]):

$$
X_{1}=\frac{\partial}{\partial y}+\bar{u}_{1} \frac{\partial}{\partial u}+f \frac{\partial}{\partial u_{1}}+D(f) \frac{\partial}{\partial u_{2}}+\ldots, X_{2}=\frac{\partial}{\partial \bar{u}_{1}} .
$$

Here $\bar{u}_{1}=u_{y}, u_{1}=u_{x}, u_{2}=u_{x x}, \ldots, f=e^{u}+e^{-u}$, an $D$ is the operator of the total differentiation w.r.t. the variable $x$. Let $L_{n}$ be a linear space of commutators making the lengths $n-1, n=2,3, \ldots$. Then the characteristic Lie ring $A$ can be represented as

$$
A=\sum_{i=2}^{\infty} L_{i} .
$$

We let

$$
\mathfrak{L}_{n}=\sum_{i=2}^{n} L_{i} .
$$

A complete description of the structure of the ring $A$ was given in [3]. In particular, the ring $A$ satisfies the formulae

$$
\begin{gathered}
\operatorname{dim} L_{n}=\left\{\begin{array}{l}
2, \quad \text { as } n=2 k, \\
1, \quad \text { as } n=2 k-1 .
\end{array} \quad k=3,4, \ldots,\right. \\
\operatorname{dim} L_{2}=2, \quad \operatorname{dim} L_{3}=1, \quad \operatorname{dim} L_{4}=1, \quad \operatorname{dim} L_{5}=1 .
\end{gathered}
$$

A.V. Zhiber, S.N. Kamaeva, Construction of exact solution to Sine-Gordon equation on the BASE OF ITS CHARACTERISTIC LIE RING.

(c) Zhiber A.V., Kamaeva S.N. 2016.

The study is supported by the grant of Russian Science Foundation (project no. 15-11-2007).

Submitted July 6, 2016. 
Exact solutions to equation (1) of $N$ th order arise if we impose the condition

$$
\operatorname{dim} \mathfrak{L}_{2 N}=\operatorname{dim} \mathfrak{L}_{2 N-1}+1
$$

on the space $\mathfrak{L}_{2 N}$.

In the work we study the case $N=3$. In the general situation $\operatorname{dim} \mathfrak{L}_{6}=7$ and the linear space $\mathfrak{L}_{6}$ is generated by the vector fields $X_{1}, X_{2}, X_{3}=\left[X_{2}, X_{1}\right], X_{5}=\left[X_{1}, X_{3}\right], X_{7}=\left[X_{1}, X_{5}\right]$, $X_{8}=\left[X_{2}, X_{7}\right], X_{9}=\left[X_{1}, X_{7}\right]$. At that, $\operatorname{dim} \mathfrak{L}_{i}=i, i=2,3,4,5$, and the formulae

$$
\begin{array}{ll}
{\left[D, X_{3}\right]=-f^{\prime} X_{2},} & {\left[D, X_{5}\right]=f^{\prime} X_{3}-f X_{1},} \\
{\left[D, X_{7}\right]=f^{\prime} X_{5},} & {\left[D, X_{8}\right]=f X_{5}, \quad\left[D, X_{9}\right]=-f X_{8}+f^{\prime} X_{7},}
\end{array}
$$

hold true, where $D$ is the operator of the total differentiation w.r.t. $x$. The total description of finding these values can be found in the work [3].

We study two cases, $\operatorname{dim} \mathfrak{L}_{6}=6$ and $\operatorname{dim} \mathfrak{L}_{6}=5$.

\section{Characteristic Lie Ring in the Case $\operatorname{dim} \mathfrak{L}_{6}=6$}

In this section we obtain the conditions for the solutions to the Sine-Gordon equation, when $\operatorname{dim} \mathfrak{L}_{6}=6$, that is, $\operatorname{dim} L_{6}=1$. Namely, we consider two cases:

1) The vector fields $X_{1}, X_{2}, X_{3}, X_{5}, X_{7}, X_{9}$ are linearly independent and $X_{8}$ is their linear combination.

2) The vector fields $X_{1}, X_{2}, X_{3}, X_{5}, X_{7}, X_{8}$ are linear independent and $X_{9}$ is their linear combination.

Assume the first case holds true, that is,

$$
X_{8}=\alpha_{1} X_{1}+\alpha_{2} X_{3}+\alpha_{3} X_{5}+\alpha_{4} X_{7}+\alpha_{5} X_{9} .
$$

Then

$$
\left[D, X_{8}\right]=\left[D, \alpha_{1} X_{1}+\alpha_{2} X_{3}+\alpha_{3} X_{5}+\alpha_{4} X_{7}+\alpha_{5} X_{9}\right]
$$

By the property of commutators, the latter relation can be written as

$$
\begin{aligned}
{\left[D, X_{8}\right]=} & \alpha_{1}\left[D, X_{1}\right]+D\left(\alpha_{1}\right) X_{1}+\alpha_{2}\left[D, X_{3}\right]+D\left(\alpha_{2}\right) X_{3}+\alpha_{3}\left[D, X_{5}\right]+D\left(\alpha_{3}\right) X_{5} \\
& +\alpha_{4}\left[D, X_{7}\right]+D\left(\alpha_{4}\right) X_{7}+\alpha_{5}\left[D, X_{9}\right]+D\left(\alpha_{5}\right) X_{9} .
\end{aligned}
$$

Employing formulate (2), we obtain

$$
\begin{aligned}
{\left[D, X_{8}\right]=} & -\alpha_{1} f X_{2}+D\left(\alpha_{1}\right) X_{1}-\alpha_{2} f^{\prime} X_{2}+D\left(\alpha_{2}\right) X_{3}+\alpha_{3} f^{\prime} X_{3}-\alpha_{3} f X_{1} \\
& +D\left(\alpha_{3}\right) X_{5}+\alpha_{4} f^{\prime} X_{5}+D\left(\alpha_{4}\right) X_{7}-\alpha_{1} \alpha_{5} f X_{1}-\alpha_{2} \alpha_{5} f X_{3}-\alpha_{3} \alpha_{5} f X_{5} \\
& -\alpha_{4} \alpha_{5} f X_{7}-\alpha_{5} \alpha_{5} f X_{9}+\alpha_{5} f^{\prime} X_{7}+D\left(\alpha_{5}\right) X_{9} .
\end{aligned}
$$

On the other hand,

$$
\left[D, X_{8}\right]=f X_{5},
$$

and this is why we can equate the coefficients at the independent operators $X_{i}$ and to obtain the system of the equations

$$
\begin{aligned}
D\left(\alpha_{1}\right)-\alpha_{3} f-\alpha_{1} \alpha_{5} f & =0, \\
-\alpha_{1} f-\alpha_{2} f^{\prime} & =0 \\
D\left(\alpha_{2}\right)+\alpha_{3} f^{\prime}-\alpha_{2} \alpha_{5} f & =0 \\
D\left(\alpha_{3}\right)+\alpha_{4} f^{\prime}-\alpha_{3} \alpha_{5} f & =f, \\
D\left(\alpha_{4}\right)+\alpha_{5} f^{\prime}-\alpha_{4} \alpha_{5} f & =0 \\
D\left(\alpha_{5}\right)-\alpha_{5} \alpha_{5} f & =0 .
\end{aligned}
$$

Hence, the following statement holds. 
Lemma 1. If $X_{1}, X_{2}, X_{3}, X_{5}, X_{7}, X_{9}$ are linearly independent, and $X_{8}$ is their linear combination, then relations (3)-(8) hold true.

Let us study system of equations (3)-(8). If $\alpha_{5}=0$, these equations are rewritten as

$$
\begin{aligned}
D\left(\alpha_{1}\right)-\alpha_{3} f & =0, \\
-\alpha_{1} f-\alpha_{2} f^{\prime} & =0, \\
D\left(\alpha_{2}\right)+\alpha_{3} f^{\prime} & =0, \\
D\left(\alpha_{3}\right)+\alpha_{4} f^{\prime} & =f, \\
D\left(\alpha_{4}\right) & =0 .
\end{aligned}
$$

It is easy to check that the case $\alpha_{1} \alpha_{2}=0$ can not be realized. Assume that $\alpha_{1} \alpha_{2} \neq 0$. We express $\alpha_{2}$ in equation (10) in terms of $\alpha_{1}$ :

$$
\alpha_{2}=-\frac{\alpha_{1} f}{f^{\prime}}
$$

and we differentiate this identity. Taking into consideration that $1-\frac{f^{2}}{f^{\prime 2}}=-\frac{4}{f^{\prime 2}}$, we get

$$
D\left(\alpha_{2}\right)=-D\left(\alpha_{1}\right) \frac{f}{f^{\prime}}+\frac{4 u_{1} \alpha_{1}}{f^{\prime 2}} .
$$

Taking into consideration the latter identity and relations (9) and (11), we obtain that

$$
\alpha_{3}=\frac{u_{1} \alpha_{1}}{f^{\prime}} .
$$

Now by (15) and (9) we obtain the differential equation

$$
\frac{D\left(\alpha_{1}\right)}{\alpha_{1}}=\frac{u_{1} f}{f^{\prime}}
$$

whose solution is of the form

$$
\alpha_{1}=\phi(y) f^{\prime} .
$$

Hence, employing formulae (14) and (15), we find:

$$
\begin{aligned}
& \alpha_{2}=-\phi(y) f . \\
& \alpha_{3}=u_{1} \phi(y) .
\end{aligned}
$$

It follows from equation (12) that

$$
\alpha_{4}=\frac{f-u_{2} \phi(y)}{f^{\prime}} .
$$

It remains to show that $D\left(\alpha_{4}\right)=0$. In order to do it, we need to solve the following equation

$$
D\left(\frac{f-u_{2} \phi(y)}{f^{\prime}}\right)=0 \text {. }
$$

We differentiate it and find:

$$
\phi(y)=-\frac{4 u_{1}}{u_{3} f^{\prime}-u_{1} u_{2} f} .
$$

Since $D(\phi(y))=0$, by applying the operator $D$ to the right hand side of (20) we obtain

which holds only if

$$
\frac{u_{1} u_{4} f^{\prime}-u_{2} u_{3} f^{\prime}-u_{1}^{3} u_{2} f^{\prime}}{u_{3}^{2} f^{\prime 2}-2 u_{1} u_{2} u_{3} f^{2}+u_{1}^{2} u_{2} f^{2}}=0,
$$

$$
u_{1} u_{4}-u_{2} u_{3}-u_{1}^{3} u_{2}=0 .
$$

Thus, relations (16)-(21) yields the following statement. 
Theorem 1. If $\alpha_{5}=0$, then the solution to system of equations (3)-(8) is of the form:

$$
\alpha_{1}=\frac{-4 u_{1} f^{\prime}}{u_{3} f^{\prime}-u_{1} u_{2} f}, \quad \alpha_{2}=\frac{4 u_{1} f}{u_{3} f^{\prime}-u_{1} u_{2} f}, \quad \alpha_{3}=\frac{-4 u_{1}^{2}}{u_{3} f^{\prime}-u_{1} u_{2} f}, \quad \alpha_{4}=\frac{u_{3} f-u_{1} u_{2} f^{\prime}}{u_{3} f^{\prime}-u_{1} u_{2} f},
$$

and identity (21) holds true.

The case $\alpha_{4}=0$ leads us to the relation

$$
u_{3} f-u_{1} u_{2} f^{\prime}=0,
$$

whose solution, by equation (1), is of the form:

$$
u=u(x+y) .
$$

Consider equation (21). In order to do it, we rewrite it as

$$
u_{4}=\frac{u_{2} u_{3}}{u_{1}}+u_{1}^{2} u_{2},
$$

and apply operator $\bar{D}$ due to equation (1).

If we write each derivative separately,

$$
\begin{aligned}
& \bar{D} u_{1}=u_{x} y=e^{u}+e^{-u}, \\
& \bar{D} u_{2}=D f=\left(e^{u}-e^{-u}\right) u_{1}, \\
& \bar{D} u_{3}=D^{2} f=\left(e^{u}+e^{-u}\right) u_{1}^{2}+\left(e^{u}-e^{-u}\right) u_{2}, \\
& \bar{D} u_{4}=D^{3} f=\left(e^{u}-e^{-u}\right) u_{1}^{3}+\left(e^{u}-e^{-u}\right) u_{3}+3\left(e^{u}+e^{-u}\right) u_{1} u_{2},
\end{aligned}
$$

then the right hand side of equation (23) casts into the form:

$$
u_{3}\left(e^{u}-e^{-u}\right)+\frac{u_{2}^{2}}{u_{1}}\left(e^{u}-e^{-u}\right)+u_{1}^{3}\left(e^{u}-e^{-u}\right)+3 u_{1} u_{2}\left(e^{u}+e^{-u}\right)-\frac{u_{2} u_{3}}{u_{1}^{2}}\left(e^{u}+e^{-u}\right) .
$$

We equate the latter relation to (24) and after some simple transformations we obtain

$$
u_{3}=u_{1} u_{2} \frac{e^{u}-e^{-u}}{e^{u}+e^{-u}}
$$

Equation (25) coincides with (22). Thus, a solution $u(x, y)$ to equation (25) satisfying equation (1) is of the form:

that is,

$$
u=u(x, y)
$$

$$
u^{\prime \prime}=f(u) .
$$

It is easy to show that the solutions to equation (26) satisfy equations (22) and (23). Thus, in the first case 1), under the condition $\alpha_{5}=0$, the solutions to the Sine-Gordon equation (1) are determined by equation (26).

Suppose that $\alpha_{5} \neq 0$, then by equation (8) we obtain that

$$
\alpha_{5}=\frac{1}{P(y)-\bar{u}_{1}}
$$

where $P(y)$ is a function depending on $y$.

If $\alpha_{1} \alpha_{2} \alpha_{3}=0$, then by (3)-(7) we obtain that $\alpha_{4}=\frac{f}{f^{\prime}}$. Since $D\left(\alpha_{4}\right)=-4 \frac{u_{1}}{f^{\prime 2}}$, relation (7) can be rewritten as

Therefore, we have the identity

$$
\frac{-4 u_{1}\left(P(y)-\bar{u}_{1}\right)+f^{\prime 3}-f^{2} f^{\prime}}{\left(P(y)-\bar{u}_{1}\right) f^{\prime 2}}=0 .
$$

$$
f^{\prime}+u_{1}\left(P(y)-\bar{u}_{1}\right)=0 .
$$

It is easy to show that in this case $u_{2}=0$. It implies that $u=$ const. 
We proceed to the case $\alpha_{1} \alpha_{2} \alpha_{3} \neq 0$. If we express $\alpha_{2}$ and $\alpha_{3}$ in terms of $\alpha_{1}$, we get the same relations (14) and (15). Substituting them into (3), we obtain the differential equation

Its solution is of the form

$$
\frac{D\left(\alpha_{1}\right)}{\alpha_{1}}=\frac{u_{1} f}{f^{\prime}}+\frac{f}{P(y)-\bar{u}_{1}} .
$$

$$
\alpha_{1}=\frac{\left.c_{1}(y) f^{\prime}\right)}{P(y)-\bar{u}_{1}}
$$

Therefore, formulae (14) and (15) are rewritten as

$$
\alpha_{2}=-\frac{c_{1}(y) f}{P(y)-\bar{u}_{1}}, \alpha_{3}=\frac{u_{1} c_{1}(y)}{P(y)-\bar{u}_{1}} .
$$

It remains to find $\alpha_{4}$ by equation (6)

$$
\alpha_{4}=\frac{f}{f^{\prime}}-\frac{u_{2} c_{1}(y)}{\left(P(y)-\bar{u}_{1}\right) f^{\prime}} .
$$

We proceed to equation (7). We calculate

$$
D\left(\alpha_{4}\right)=\frac{-4 u_{1}}{f^{\prime 2}}-\frac{u_{3} c_{1}(y)}{\left(P(y)-\bar{u}_{1}\right) f^{\prime}}+\frac{u_{1} u_{2} f c_{1}(y)}{\left(P(y)-\bar{u}_{1}\right) f^{\prime 2}}-\frac{u_{2} f c_{1}(y)}{\left(P(y)-\bar{u}_{1}\right) f^{\prime}}
$$

and substitute the obtained result into $(7)$. It yields the relation

$$
c_{1}(y)\left(u_{1} u_{2} f-u_{3} f^{\prime}\right)=4 f^{\prime}+4 u_{1}\left(P(y)-\bar{u}_{1}\right) .
$$

Let $c_{1}(y)=0$ or $u_{1} u_{2} f-u_{3} f^{\prime}=0$, then $\alpha_{1}=\alpha_{2}=\alpha_{3}=0, \alpha_{4}=\frac{f}{f^{\prime}}$, and

$$
P(y)=\bar{u}_{1}-\frac{f^{\prime}}{u_{1}}
$$

holds true. But since

$$
D(P(y))=\frac{u_{2} f^{\prime}}{u_{1}^{2}}=0
$$

this case can not be realized and it follows that $u_{2}=0$.

We consider the other case, that is, let $c_{1}(y)$ and $u_{1} u_{2} f-u_{3} f^{\prime}$ do not vanish simultaneously. Then it follows from the properties of the characteristic ring $x$ that $\alpha_{i}$ can depend only on the derivatives of the function $u$ w.r.t. the variable $x$ and this is why a solution exists if and only if

$$
P(y)-\bar{u}_{1}=B\left(u, u_{1}, u_{2}, \ldots\right) .
$$

We substitute (32) into formula (31) and find $c_{1}(y)$ :

$$
c_{1}(y)=\frac{4 f^{\prime}+4 u_{1} B}{u_{1} u_{2} f-u_{3} f^{\prime}} .
$$

Using that $D\left(c_{1}(y)\right)=0$, we differentiate the latter identity and find $B$, that is,

$$
B\left(u_{1} u_{4}-u_{2} u_{3}-u_{1}^{3} u_{2}\right)-u_{1}^{2} u_{2} f^{\prime}+u_{4} f^{\prime}-u_{2}^{2} f=0 .
$$

Thus, if $u_{1} u_{4}-u_{2} u_{3}-u_{1}^{3} u_{2} \neq 0$, we can find $B$ and apply the operator of differentiation $D$. Then taking into consideration that

$$
D(B)=-f
$$

we obtain the ordinary differential equation of the form

$$
u_{2} u_{5}-u_{3} u_{4}-3 u_{1} u_{2}^{3}=0 .
$$

It remains to consider the case $\alpha_{4}=0$. It is easy to check that then formulae (28), (29) and the following identity

$$
u_{2} c_{1}(y)-f\left(P(y)-\bar{u}_{1}\right)=0
$$


hold true. Let us study the latter. In order to do it, we find $c_{1}(y)$ and differentiate. As the result, we get

$$
B\left(u_{1} u_{2} f^{\prime}-u_{3} f\right)=u_{2} f^{2} .
$$

Employing this relation, it is easy to show that equation (8) does not hold. This is why that case $\alpha_{4}=0$ can not be realized.

Thus, we have proved the following theorem.

Theorem 2. If $\alpha_{5} \neq 0$, then a solution to system of equations (3)-(8) is of the form

$$
\begin{aligned}
& \alpha_{1}=\frac{4 u_{2} f^{\prime}}{u_{1}^{2} u_{2} f^{\prime}-u_{4} f^{\prime}+u_{2}^{2} f}, \quad \alpha_{2}=\frac{-4 u_{2} f}{u_{1}^{2} u_{2} f^{\prime}-u_{4} f^{\prime}+u_{2}^{2} f}, \quad \alpha_{3}=\frac{4 u_{1} u_{2}}{u_{1}^{2} u_{2} f^{\prime}-u_{4} f^{\prime}+u_{2}^{2} f}, \\
& \alpha_{4}=\frac{u_{1}^{2} u_{2} f-u_{4} f+u_{2}^{2} f^{\prime}}{u_{1}^{2} u_{2} f^{\prime}-u_{4} f^{\prime}+u_{2}^{2} f}, \quad \alpha_{5}=\frac{u_{1} u_{4}-u_{2} u_{3}+u_{1}^{3} u_{2}}{u_{1}^{2} u_{2} f^{\prime}-u_{4} f^{\prime}+u_{2}^{2} f}
\end{aligned}
$$

and relation (34) holds true.

We consider the problem on constructing a solution to system of equations (34), (1). We assume that the condition

$$
u_{1} u_{4}-u_{2} u_{3}-u_{1}^{3} u_{2} \neq 0
$$

holds true. Excluding the case

$$
u_{2}=0
$$

equation (34) can be written as

$$
D\left(\frac{u_{4}}{u_{2}}\right)-\frac{3}{2} D\left(u_{1}^{2}\right)=0
$$

that is,

$$
u_{4}=\frac{3}{2} u_{1}^{2} u_{2}+\psi(y) u_{2}
$$

Here $\psi(y)$ is an arbitrary function. Thus, equations (34) and (37) are equivalent. We note that the solutions of equation (36) satisfy both equations (34) and (37) and at the same time, condition (35) fails.

We apply the operator $\bar{D}$ to the both sides of equation (37) and taking into consideration the formulae

$$
\begin{aligned}
& D \bar{D} u=e^{u}+e^{-u}, \\
& D^{2} \bar{D} u=\left(e^{u}-e^{-u}\right) u_{1}, \\
& D^{3} \bar{D} u=\left(e^{u}+e^{-u}\right) u_{1}^{2}+\left(e^{u}-e^{-u}\right) u_{2}, \\
& D^{4} \bar{D} u=\left(e^{u}+e^{-u}\right) u_{1}^{3}+\left(e^{u}-e^{-u}\right) u_{3}+3\left(e^{u}+e^{-u}\right) u_{1} u_{2},
\end{aligned}
$$

we get

$$
u_{3}=\frac{1}{2} u_{1}^{3}+\frac{\psi^{\prime}(y) u_{2}}{e^{u}-e^{-u}}+\psi(y) u_{1} .
$$

We note that solution $u$ to system (34), (1) satisfies equation (38). On the other hand, equation (37) is equivalent to the equation

$$
u_{3}=\frac{1}{2} u_{1}^{3}+\psi(y) u_{1}+h(y)
$$

Thus, solution $u$ to system (34), (1) satisfies simultaneously equations (38) and (39). Therefore, this solution satisfies the equation

$$
\frac{\psi^{\prime}(y) u_{2}}{e^{u}-e^{-u}}=h(y)
$$


If $\psi(y) \neq 0$, then

$$
u_{2}=\frac{h(y)}{\psi^{\prime}(y)}\left(e^{u}-e^{-u}\right)
$$

and therefore,

$$
u_{1}^{2}=2 \frac{h(y)}{\psi^{\prime}(y)}\left(e^{u}+e^{-u}\right)+\omega(y) .
$$

On the other hand, it follows from (41) that

$$
u_{3}=\frac{h(y)}{\psi^{\prime}(y)}\left(e^{u}+e^{-u}\right) u_{1}
$$

We substitute derivatives (42) and (43) into equation (39) to obtain

$$
\left(\frac{1}{2} \omega(y)+\psi(y)\right) u_{1}+h(y)=0
$$

If $\frac{1}{2} \omega(y)+\psi(y)=0$, it follows from (44) that $h(y)=0$ and by (41) we have (36). If $\frac{1}{2} \omega(y)+$ $\psi(y) \neq 0$, by $(44)$ we obtain that

$$
u_{1}=-\frac{h(y)}{\frac{1}{2} \omega(y)+\psi(y)},
$$

and therefore, we arrive at equation (36).

If $\psi^{\prime}(y)=0$, then $\psi(y) \equiv c_{2}(y)$, where $c_{2}(y)$ is a constant, and by (40) we obtain that $h(y)=0$. Then equations (38) and (39) become

$$
u_{3}=\frac{1}{2} u_{1}^{3}+c_{2}(y) u_{1}
$$

We calculate expression (35):

$$
\left(\frac{3}{2} u_{1}^{2} u_{2}+c_{2}(y)\right) u_{1}-u_{2}\left(\frac{1}{2} u_{1}^{3}+c_{2}(y) u_{1}\right)-u_{1}^{3} u_{2}=0
$$

Hence, condition (35) fails. Therefore, the case $\alpha_{5} \neq 0$ can not be realized.

We proceed to case 2). Here

$$
X_{9}=\beta_{1} X_{1}+\beta_{2} X_{3}+\beta_{3} X_{5}+\beta_{4} X_{7}+\beta_{5} X_{8} .
$$

As in the first case, we obtain the system

$$
\begin{aligned}
& D\left(\beta_{1}\right)-\beta_{3} f=0, \\
& -\beta_{1} f-\beta_{2} f^{\prime}=0, \\
& D\left(\beta_{2}\right)+\beta_{3} f^{\prime}=0, \\
& D\left(\beta_{3}\right)+\beta_{4} f^{\prime}+\beta_{5} f=0, \\
& D\left(\beta_{4}\right)=f^{\prime}, \\
& D\left(\beta_{5}\right)=-f .
\end{aligned}
$$

Let us show that this case can be reduced to the previous one. It is easy to see that $\beta_{5} \neq 0$ in equation (51). Therefore, $X_{8}$ can be written as

$$
X_{8}=-\frac{\beta_{1}}{\beta_{5}} X_{1}-\frac{\beta_{2}}{\beta_{5}} X_{3}-\frac{\beta_{3}}{\beta_{5}} X_{5}-\frac{\beta_{4}}{\beta_{5}} X_{7}+\frac{1}{\beta_{5}} X_{9}
$$

Assume that $X_{1}, X_{2}, X_{3}, X_{5}, X_{7}, X_{9}$ are linearly dependent in relation (52) and $X_{9}$ can be expressed in terms of $X_{1}, X_{2}, X_{3}, X_{5}, X_{7}$. It means that $X_{8}$ can be also expressed in terms of these functions. Therefore, $X_{1}, X_{2}, X_{3}, X_{5}, X_{7}, X_{8}$ are linearly dependent that contradicts the given condition. 


\section{Characteristic Lie Ring in the CaSe dim $\mathfrak{L}_{6}=5$}

In this section we study the solution to the Sine-Gordon equation, whose algebra is finitedimensional and its dimension is 5 . That is, we consider the case, when $X_{1}, X_{2}, X_{3}, X_{5}, X_{7}$ are linearly independent and $X_{8}$ and $X_{9}$ are their linear combinations:

$$
X_{9}=\lambda_{1} X_{1}+\lambda_{2} X_{3}+\lambda_{3} X_{5}+\lambda_{4} X_{7}
$$

and

$$
X_{8}=\mu_{1} X_{1}+\mu_{2} X_{3}+\mu_{3} X_{5}+\mu_{4} X_{7}
$$

Then the identities

$$
\left[D, X_{9}\right]=\left[D, \lambda_{1} X_{1}+\lambda_{2} X_{3}+\lambda_{3} X_{5}+\lambda_{4} X_{7}\right]=f X_{5}
$$

and

hold true.

$$
\left[D, X_{8}\right]=\left[D, \mu_{1} X_{1}+\mu_{2} X_{3}+\mu_{3} X_{5}+\mu_{4} X_{7}\right]=-f X_{8}+f^{\prime} X_{7}
$$

Employing formulae (2), we expand commutators and obtain the system of equations:

$$
\begin{aligned}
& D\left(\lambda_{1}\right)-\lambda_{3} f=0, \\
& -\lambda_{1} f-\lambda_{2} f^{\prime}=0, \\
& D\left(\lambda_{2}\right)+\lambda_{3} f^{\prime}=0, \\
& D\left(\lambda_{3}\right)+\lambda_{4} f^{\prime}=f, \\
& D\left(\lambda_{4}\right)=0, \\
& D\left(\mu_{1}\right)-\mu_{3} f=-\lambda_{1} f, \\
& -\mu_{1} f-\mu_{2} f^{\prime}=0, \\
& D\left(\mu_{2}\right)+\mu_{3} f^{\prime}=-\lambda_{2} f, \\
& D\left(\mu_{3}\right)+\mu_{4} f^{\prime}=-\lambda_{3} f, \\
& D\left(\mu_{4}\right)=-\lambda_{4} f+f^{\prime} .
\end{aligned}
$$

It is easy to see that equations (53)-(57) coincide with equations (9)-(13). Thus, they satisfy the assumptions of Theorem 1. This is why we can assume that the coefficients $\lambda_{i}$ are found. Let us find $\mu_{i}, i=1, \ldots, 4$.

By formulae (59) and (60) we write

$$
\begin{gathered}
\mu_{2}=-\frac{\mu_{1} f}{f^{\prime}}, \\
\mu_{3}=\frac{\mu_{1} u_{1}}{f^{\prime}} .
\end{gathered}
$$

We substitute (64) into (58). We obtain an inhomogeneous differential equation of the form

$$
D\left(\mu_{1}\right)-u_{1} \mu_{1} \frac{f}{f^{\prime}}=-f f^{\prime} \varphi(y)
$$

whose solution is given by the formula

$$
\mu_{1}=f^{\prime} c(y)-\bar{u}_{1} f \varphi(y) .
$$

Then by formulae (61), (63) and (64) we can find other coefficients. Namely,

$$
\begin{aligned}
& \mu_{2}=-f c(y)-\bar{u}_{1} f \varphi(y), \\
& \mu_{3}=u_{1} c(y)-u_{1} \bar{u}_{1} f \varphi(y), \\
& \mu_{4}=\frac{\bar{u}_{1} u_{2} \varphi(y)-u_{2} c(y)}{f^{\prime}} .
\end{aligned}
$$


In view of formulae (19), (67), equation (62) becomes

$$
c(y)=\bar{u}_{1} \varphi(y)+\frac{4 f^{\prime}}{u_{3} f^{\prime}-u_{1} u_{2} f} .
$$

Let us study all possible cases of its solutions. It is easy to show that the condition $c(y)=0$ implies that $u_{2}=0$. If $c(y) \neq 0$, taking into consideration equation (21), we obtain

$$
u_{2}^{2} f+u_{1}^{2} u_{2} f^{\prime}-u_{4} f^{\prime}=0 .
$$

Now by (21) and (68) we have

$$
u_{3} f^{\prime}-u_{1} u_{2} f=0 .
$$

The latter does not satisfied. Thus, we following statement holds.

Lemma 2. If the dimension of the characteristic Lie ring is equal to 5 , then the Sine-Gordon equation has no solution.

\section{BIBLIOGRAPHY}

1. S. Novikov, S.V. Manakov, L.P. Pitaevskii, V.E. Zakharov. Theory of solitons. The inverse scattering method. Nauka, Moscow (1980). [Contemp. Soviet Math. Plenum Publishing Corporation, New York (1984).]

2. L.D. Faddeev, L.A. Takhtadzhyan. Hamiltonian methods in the theory of solitons. Nauka, Moscow (1986). [Springer Series in Soviet Mathematics. Springer, Berlin (1987).]

3. A.V. Zhiber, R.D. Murtazina, I.T. Habibullin, A.B. Shabat. Characteristic Lie rings and nonlinear integrable equations. Insitut Kompyut. Issled. Izhevsk (2012). (in Russian).

Anatoly Vasil'evich Zhiber, Institute of Mathematics,

Ufa Scientific Center,

Russian Academy of Sciences,

Chernyshevsky str. 112,

450077, Ufa, Russia

E-mail: zhiber@mail.ru

Sabina Nazirovna Kamaeva,

Ufa State Aviation Technical University,

Karl Marx str. 12,

450000, Ufa, Russia

E-mail: sabbi@mail.ru 